\title{
Theoretical and Experimental research of pre-stressed CFRP tendon aluminum alloy beam with double struts
}

\author{
Pengcheng $\mathrm{Zhu}^{1,2, \mathrm{a}}$, Longxing $\mathrm{Xu}^{2, \mathrm{~b}}$, Chuan Liang ${ }^{2, \mathrm{c}}$, Pengcheng $\mathrm{Ai}^{2, \mathrm{~d}}$ \\ ${ }^{1}$ Institute of Battlefield Engineering, PLA University of Science and Technology, Nanjing 210007, \\ China \\ ${ }^{2}$ Wuxi First Scientific Research Institute, Wuxi 214035,China

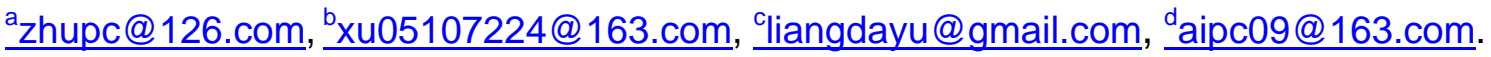

Keywords: External pre-stressing; CFRP tendon; Aluminum beam; two struts.

Abstract. The external pre-stressing technique has been commonly used in the construction field because it can effectively improve the carrying capacity and stiffness of the structure and is widely applicable for many different types of structures. In this research, the pre-stressed CFRP tendon was used to strengthen aluminum alloy beam with two struts. Theoretical and experimental methods are used to study the stress distribution of the aluminum alloy beam and CFRP tendon. The theoretical solution is very close to the experimental results, So that the theoretical method is rational. The parameter analysis of the structure is also studied. Results of the parametric analysis shows that larger pre-stressing force, higher load capacity of beam, and CFRP tendon of larger diameter, higher load capacity of beam, and effect of increasing load capacity aluminum alloy beam strengthened by pre-stressed CFRP tendon is better than steel beam.

\section{Introduction}

The external pre-stressing method, which is a post-tensioning technique, has been commonly used to strengthen old bridges and building structures. Because it provides considerable benefit in terms of easy applicability and economic feasibility. It is also used in the construction of new bridges and building structures [1]. While the pre-stressing method used for reinforced and pre-stressed concrete bridges has advanced remarkably, now it is also been widely used in steel bridge construction, steel structures are only slightly affected by friction loss when steel tendons are applied. Therefore, there are more advantages in using the external post-tensioning method to steel structures than to concrete structures [2].

The pre-stressing method for steel structures can effectively improve the carrying capacity and stiffness of the structure. The application of high performance steel tendons reduces the size and amount of steel members. Meanwhile, the total demand for steel decreases and the load-carrying capacity of structures increases [3]. Fabrication and mechanical properties of internally pre-stressed steel I beam produced by combining steel $\mathrm{T}$ profile and pre-stressing steel plate were studied, it was observed that flexural properties of pre-stressed steel I beam produced by this method has increased by about 30\% [4]. Moreover, the strengthening method that uses the post-tensioning technique can be analyzed by classical structural analysis method $[5,6,7,8]$.

CFRP has high strength, superior creep properties, low relaxations and can withstand high jacking stress, CFRP tendons can exert its high-tension strength, it has been widely used to pre-stressed concrete and steel structures [9,10]. There are reports about large, practical applications that have used external CFRP pre-stressing and post-stressing of bridges [11].

The aim of this research is to study the stress distribution of the aluminum alloy beam and CFRP tendon by theory and experiment method, and based on the theoretical method, the effect of the parameters is researched.

\section{Theoretical solution}

The force method can be used to analyze the pre-stressed CFRP tendons aluminum alloy beam with double struts. In order to calculate the increment of tension forces in CFRP tendons as the 
external loads change. A compatibility equation related to deflection can be introduced tosolve the calculation of the extension.

$$
\delta_{11} X_{1}+\Delta_{1 P}=0
$$

Where $\delta_{11}$ is the elongation length caused by the unit tendon force, $\Delta_{1 P}$ is the elongation length in the middle of beam caused by the applied loads .

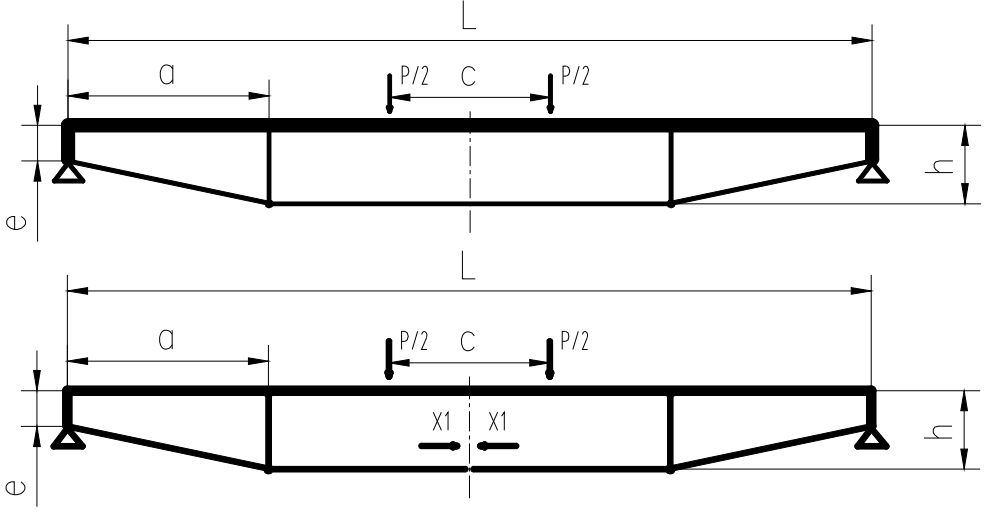

Fig. 1 diagram calculation

Equations that can be used to evaluate these elongation lengths can be deduced based on virtual work principle, as shown in Eq.2- Eq.3

$$
\begin{aligned}
& \delta_{11}=\frac{h^{2}(l-2 a)+2 a e^{2}+\frac{2}{3} a(h-e)^{2}}{E_{B} I_{B}}+\frac{l}{E_{B} A_{B}}+\frac{2\left[a^{2}+(h-e)^{2}\right]^{\frac{3}{2}}}{E_{T 1} A_{T 1} a^{2}}+\frac{(l-2 a)}{E_{T 2} A_{T 2}}+\frac{2(h-e)^{2} h}{E_{S} A_{S} a^{2}} \\
& \Delta_{1 P}=\frac{2 P}{E_{B} I_{B}}\left[\frac{1}{12} a^{2}(2 h+e)+\frac{1}{4} h\left(\frac{l-c}{2}\right)^{2}-\frac{1}{4} h a^{2}-\frac{1}{4} h(l-c) c\right] \\
& X_{1}=\frac{\Delta_{1 P}}{\delta_{11}} \\
& X_{0} \text { Initial value of pre-stressed force }
\end{aligned}
$$

Pre-stressing phase:

Stress of bottom flange: $\sigma_{02}=\frac{X_{0}}{A}+\frac{X_{0} e}{W_{2}}+\frac{X_{0}(h-e)}{W_{2}}=\frac{X_{0}}{A}+\frac{X_{0} h}{W_{2}}$

Loading phase:

Stress of top flange: $\sigma_{1}=-\frac{P(l-c)}{4 W_{1}}+\frac{\left(X_{0}+X_{1}\right) h}{W_{1}}-\frac{X_{0}+X_{1}}{A}$

Stress of bottom flange $\sigma_{1}=\frac{P(l-c)}{4 W_{2}}-\frac{\left(X_{0}+X_{1}\right) h}{W_{2}}-\frac{X_{0}+X_{1}}{A}$

Stress of side tendon : $\sigma_{T 1}=\frac{\left(\sqrt{a^{2}+(h-e)^{2}} / a\right)\left(X_{0}+X_{1}\right)}{A_{T 1}}$

Stress of middle tendon: $\sigma_{T 2}=\frac{X_{0}+X_{1}}{A_{T 2}}$

Stress of strut: $\sigma_{s}=\frac{(h-e)\left(X_{0}+X_{1}\right)}{a A_{s}}$

\section{Experimental investigation}

Design of pre-stressed beam. The way to external pre-stressing the beam is shown as Fig.2, ends of the CFRP tendon are connected with steel tubes, that are extruded to form great clamping force to anchor the CFRP tendon, the CFRP tendon is pre-stressed by two oil jack. The CFRP tendons are 
tensioned in four load steps, $0 \mathrm{kN}, 40 \mathrm{kN}, 60 \mathrm{kN}$ and $80 \mathrm{kN}$. When the CFRP tendons are tensioned, the deflection and strain of the beam are measured at every load step.

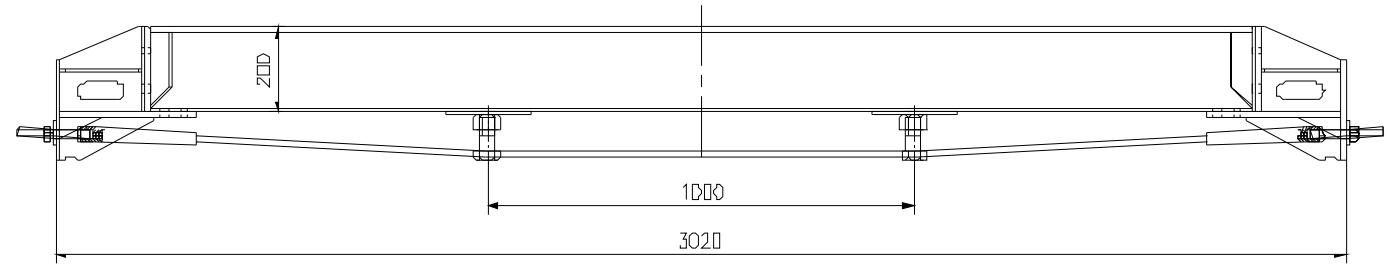

Fig.2 Prestressed beam with two struts

The upper aluminum alloy I-beam is used with a $100 \mathrm{~mm}$ wide flange, a height of $200 \mathrm{~mm}$, a 10 mm flange thickness, and a $10 \mathrm{~mm}$ web thickness. The CFRP tendon used in this study has a diameter of $12.7 \mathrm{~mm}$. The properties of the materials that were used are given in Table1

Table 1 Material properties of beam and CFRP tendon

\begin{tabular}{lcccc}
\hline Type & $\begin{array}{c}\text { Cross section area } \\
\left(\mathrm{mm}^{2}\right)\end{array}$ & $\begin{array}{c}\text { Moment of inertia } \\
\left(\mathrm{mm}^{4}\right)\end{array}$ & $\begin{array}{c}\text { Modulus of } \\
\text { elasticity }(\mathrm{GPa})\end{array}$ & $\begin{array}{c}\text { Yield strength } \\
(\mathrm{MPa})\end{array}$ \\
\hline Alu. Beam & 3800 & $2.29 \times 10^{7}$ & 70 & 228 \\
CFRP & 126 & $/$ & 124 & 1740 \\
\hline
\end{tabular}

Test method. In order to evaluate the improvement of the flexural capacity with different pre-stressing force, a four-point-loading test was conducted on aluminum alloy beams. The load is transferred from the jack to the aluminum alloy beam specimen by using a two-point loading beam. Two roller supports carried the reactions. The load span is $740 \mathrm{~mm}$. In each step, the load interval is 5 $\mathrm{kN}$. The measurement instruments included one Linear Variable Deformation Transducer (LVDT) at the mid-span to measure the deflection and one load cell to measure the applied load.

A universal static load testing machine (MTs) was used to apply load on beams, as shown in Fig. 3. In order to measure the span deflection of the aluminum alloy beam, a linear variable differential transformer (LVDT) is located in the mid-span of aluminum alloy beam, Steel strain gauges are also attached on the top flange and the bottom flange to measure the stress in the mid-span of aluminum alloy beam, as shown in Fig. 4. In addition, strain gauges are also positioned onto the surface of CFRP tendons in order to obtain the strain to loading ratio when beams are tensioned.

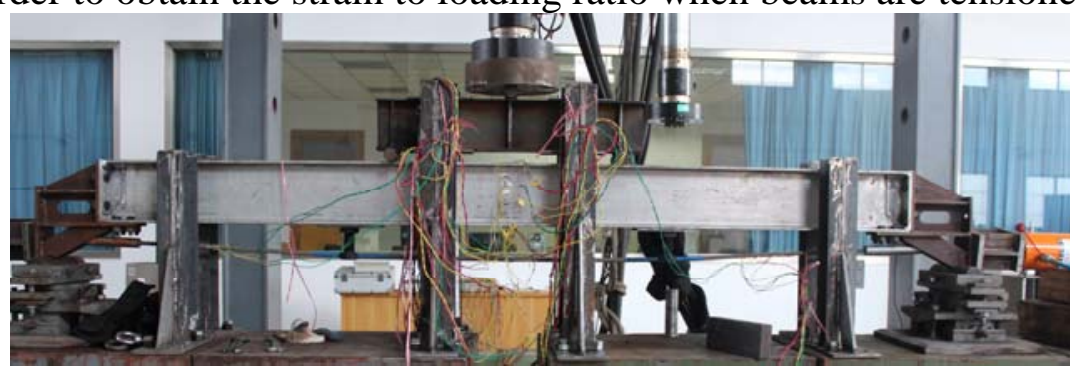

Fig. 3 Flexural test set-up

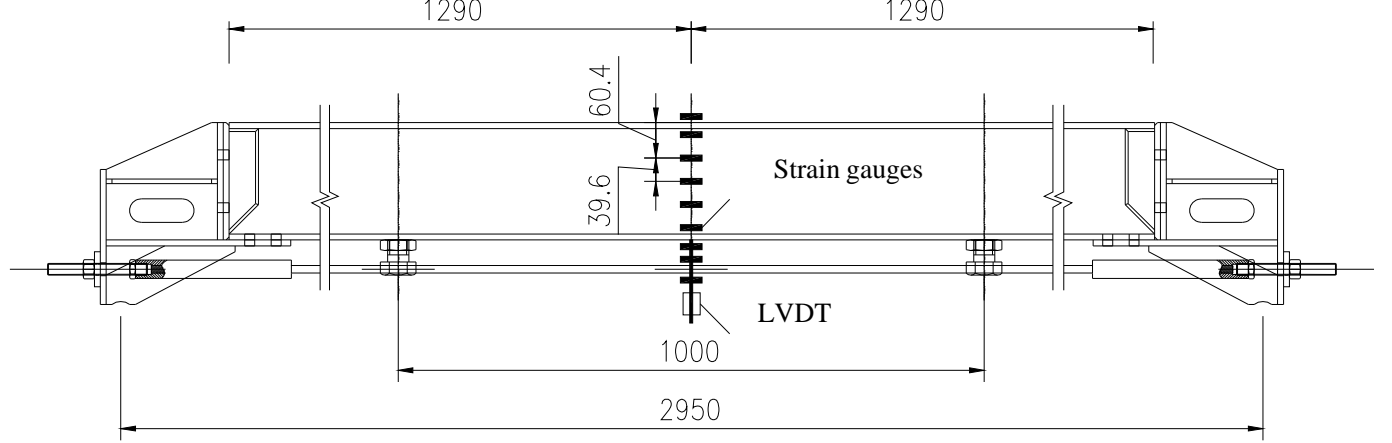

Fig. 4 Location of LVDT and strain gauges

\section{Comparison of theoretical and Experimental result}

The stress is calculated by theoretical equations and experimental test in cases of $40 \mathrm{kN}, 60 \mathrm{kN}$ and $80 \mathrm{kN}$ presented forces respectively, The comparative results between theory and experiment 
are shown in Fig.5. Theoretical and experimental results show that the bottom flange bearing pressure stress, the top flange bearing tension stress after the aluminum alloy beam was prestressed. While the stress stage changed when the beam was applied load from the jack, the bottom flange sustaining tensile stress, the top flange bearing compressive stress. The load-stress variation obtained by theoretical solution and experimental test was completely the same. And comparing the stress value of the flange by theoretical solution and experimental test, the stress value is completely the same, too. Thus the theoretical solution is correct, and it can be used to calculate the stress in construction field.

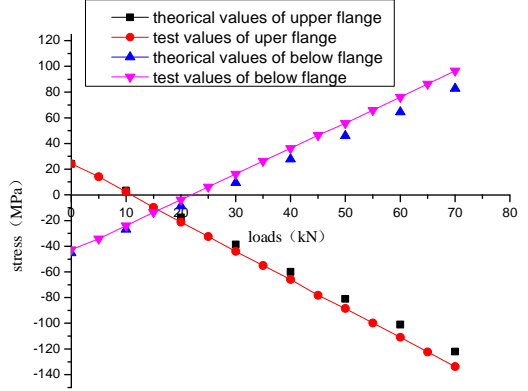

(a) The prestressed force $=40 \mathrm{kN}$

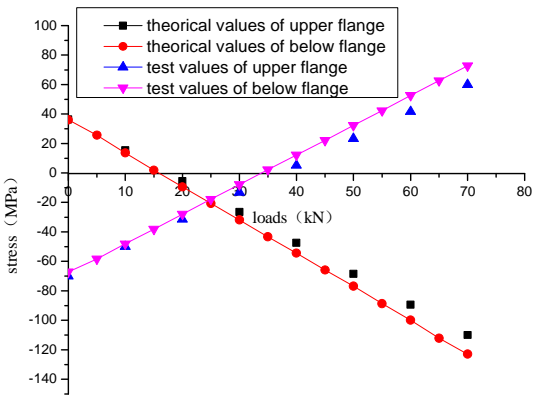

(b) The prestressed force $=60 \mathrm{kN}$

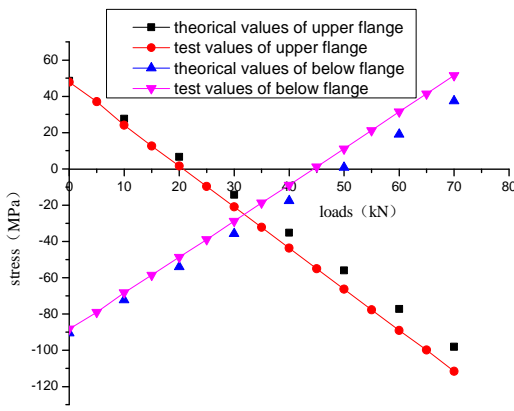

(c) The prestressed force $=80 \mathrm{kN}$

Fig. 5 comparison of theoretical solution and experimental test

\section{Parameter analysis}

Based on the theoretical solution, the stress of aluminum alloy beam with two struts is calculated with different prestressed force, different beam materials and different diameter of CFRP tendon, we can get the results that parameters influence the load-stress variation as below.

When the prestressed force is $40 \mathrm{kN}, 60 \mathrm{kN}$ and $80 \mathrm{kN}$, the top flange's stress under different load is shown in Fig.6(a). Under the same applied load, the stronger the prestressed force is, the smaller the stress is. It obviously shows that the prestressed force can improve the applied load. When we design the structure, we should consider the bottom flange's stress and the CFRP tendon's stress, which should not exceed the design value.

The beam is fabricated by aluminum and steel,when the prestressed force, the dimeter of tendons and the material of tendons are fixed, the top flange's stress of aluminum is smaller than the steel, as shown in Fig.6 (b), the results showed that the aluminum beams prestressed CFRP tendons have higher load capacity.

With the same beam section, materials, prestressed force and tendons materials, if the diameter of CFRP tendon bigger, the stress on the top flange is lower under the same loads, as shows in Fig.6(c). The results showed that the larger the diameters of CFRP tendons can obviously improver the load capability.

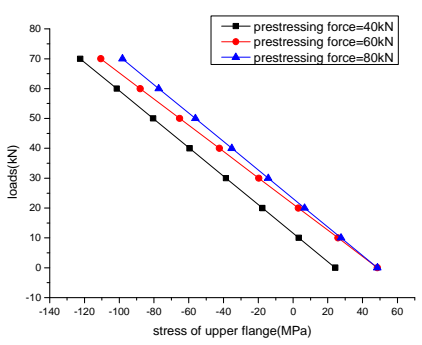

(a) prestressed force

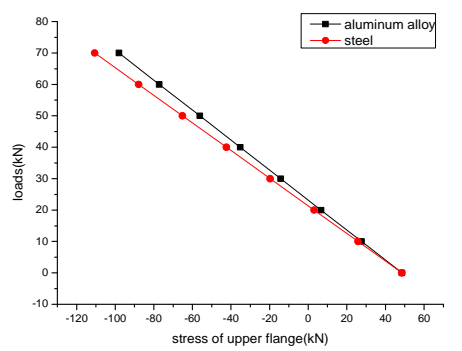

(b) materials of the beam

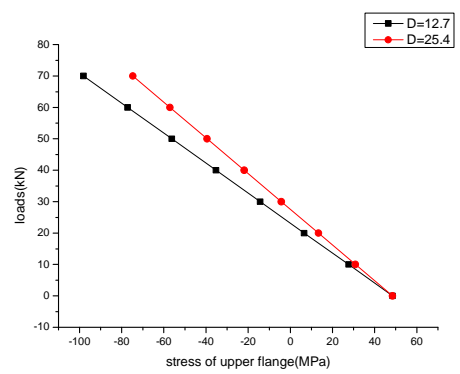

(c) diameter of CFRP tendon

Fig. 6 the parameter analysis of prestressed beam

\section{Conclusions}

The flexural behavior of external prestressing CFRP tendons aluminum alloy beam with double struts is investigated. Based on forced method, the calculation formula is derived, the double strut 
prestressing CFRP tendons aluminum alloy beam is designed and producted and prestressed beam bearing capacity test is carried out to verify the theoretical results.

Theoretical and experimental methods are used to study the stress distribution of the aluminum alloy beam and CFRP tendon. The theoretical solution is very close to the experimental results, So that the theoretical method is rational, The parametric analysis of the structure is also studied. Results of the parameter analysis shows that larger prestressing force, higher load capacity of beam, and CFRP tendon of larger diameter , higher load capacity of beam, and effect of increasing load capacity aluminum alloy beam strengthened by prestressed CFRP tendon is better than steel beam.

\section{References}

[1] Troitsky MS. Prestressed steel bridges. New York: Van nostrand Co: 1990.

[2] AASTO. Standard specification for highway bridges.1st ed.1994.

[3] Shantong Zhong. Prestressed steel structures. China:Harbin Polytechnical Inc:1986,In Chinese.

[4] Yusuf Ozcatalbas, Alpay Ozer, Material and Design 28 (2007) 1988-1993.

[5] Pengcheng Zhu, Mingkang Gou and Yinzhi Zhou, Applied Mechanics and Materials Vols.578-579(2014)pp155-159

[6] Alessandro Zona, laura Ragni, Andrea Dall'Asta. Journal of Constructional Steel Research . 65(2009)308-313.

[7] B.Belletti and A.Gasperi. Journal of Structural Engineering, Vol.136,NO.9( 2010) 1131-1139.

[8] Sunkyu Park,Taewan Kim. Journal of Constructional Steel Research . 66(2010)125-132.

[9] Li F, Zhao QL, Chen HS, et al. Compos Struct 2010; 92: 2400-2405.

[10] Jacob W.Schmidt, Anders Bennitz. Construction and Building Materials,32(2012)100-121.

[11] Grace NF, Abdel-/Sayed G. Construction. SAMPE J 2002;38(5):28-33. 